\title{
Mechanisms Controlling Accurate Changes in Elbow Torque in Humans
}

\author{
Paul J. Cordo \\ Neurological Sciences Institute of Good Samaritan Hospital and Medical Center, Portland, Oregon 97209
}

This paper addresses a fundamental question of how motor commands specify target torque levels. Human subjects produced fast and accurate changes in torque with the isometric elbow joint. Visual stimuli were used to indicate target torque levels as well as to cue subjects to initiate their responses. During rapid changes in torque from one steadystate level to another, target torque was achieved through a sequence of approximations. During the first $200-250$ msec of responses produced in the presence of visual feedback, 3 distinct control mechanisms were recruited to guide torque to the target level.

The timing and accuracy of each control mechanism were evaluated. The first control mechanism was triggered by the visual stimulus and produced the initial rise in torque. Target torque predictability was found to strongly influence the accuracy of this control mechanism. The second control mechanism produced a corrective adjustment in torque within roughly the first 100 msec of responses. This mechanism incorporated target torque information provided by the stimulus into the response. The third control mechanism began 200-250 msec after response onset and produced corrective adjustments based on visual feedback of torque errors.

The stability of the visual feedback mechanism was evaluated because of a long loop delay. Two strategies were used to control stability: low gain and information transfer between the visual feedback mechanism and the preceding (second) control mechanism.

A central issue in the study of goal-directed motor activity is how motor commands specify the final position or torque. Most studies have presumed that the final position or torque is encoded in early stages of motor commands. For example, the "equilibrium point hypothesis" of limb control holds that the initial activation levels of opposing muscle groups specify the final joint position by setting muscle stiffness (Feldman, 1966a, b; Polit and Bizzi, 1979) and that the net stiffness of the joint uniquely determines the final (equilibrium) limb position. Im-

\footnotetext{
Received Jan. 13 1986; revised Apr. 30, 1986; accepted Aug. 11, 1986.

The assistance with experiments and data analysis by Colleen MacIntosh is greatly appreciated. This work was supported by United States Public Health Service Grant AN31017 and grants from The Medical Research Foundation of Oregon, the Collins Medical Trust, and BRSG S07 RR05593.

Correspondence should be addressed to Dr. Paul J. Cordo, Neurological Sciences Institute of Good Samaritan Hospital and Medical Center, 1120 N.W. 20th Avenue, Portland, OR 97209.

Copyright (C) 1987 Society for Neuroscience $0270-6474 / 87 / 020432-11 \$ 02.00 / 0$
}

pulse variability models (Schmidt et al., 1979) also imply that the final position is specified early in the motor command since these models are based on open-loop, impulse control of limb position. Other studies have examined EMG patterns associated with rapid limb movements to determine how these patterns are related to the final position. During rapid and accurate movement, agonist and antagonist muscles are characteristically activated with bursts of EMG (Wachholder and Altenburger, 1926; Hallett et al., 1975). Examinations of the amplitude and the timing of early EMG bursts have revealed complex relationships between EMG patterns and the final limb position (Ghez and Vicario, 1978; Brown and Cooke, 1981, 1984).

Recent experimental findings conflict with the view that early stages of motor commands are the primary determinant of the final limb position. The equilibrium point hypothesis predicts that the motor command producing a given final limb position is independent of initial position. However, rapid movements from different initial positions to the same target are associated with different patterns of muscular activity (Sittig et al., 1985). Furthermore, tonic EMG levels responsible for final limb position in moderate velocity movements are attained only after $400 \mathrm{msec}$ (Bizzi and Abend, 1983). In other words, the final limb position is partly specified by later stages of the motor command. This late specification helps to explain why the relationship between early EMG activity and the final limb position or force is so complex (Ghez and Vicario, 1978; Brown and Cooke, 1981, 1984).

An alternate view of limb control is that the limb attains the final position through a process of successive approximation rather than through early specification. Studies attempting to explain the basis of Fitt's Law (Fitts, 1954) proposed that the final limb position was reached through a process of iterative correction of response errors by the same feedback mechanism (Crossman and Goodeve, 1963; Keele, 1968). Greene (1972, 1982) has theorized that trajectory control could be simplified by updating motor responses with a number of different control mechanisms.

This paper addresses the question of how motor commands specify the final joint torque. In the experiments described below, subjects produced fast and accurate changes in torque with the isometric elbow joint. The experimental paradigm was designed to induce torque responses with large initial errors to prolong the process of target acquisition. Target torque level predictability and visual feedback were experimentally controlled to identify discrete control mechanisms responsible for target acquisition. Preliminary accounts of these experiments have been reported previously (Cordo and Nashner, 1981; Cordo, 1983). 


\section{Materials and Methods}

\section{Apparatus}

Adult human subjects produced torque at the elbow joint in response to visually presented cues. Subjects sat at a table and placed their right wrist in a loosely fitting cuff. The forearm was oriented horizontally above the table and extended $30^{\circ}$ off a line parallel to the chest. The elbow rested in a 3-cm-deep foam cavity that rotated freely. A padded, cupped frame oriented $30^{\circ}$ from the horizontal supported the upper arm from behind. Subjects leaned into a padded chest support. These supports and braces constrained the arm such that torque exerted on the cuff by the wrist was produced entirely by the elbow joint musculature.

The wrist cuff was attached to a rigid, isometric support (stiffness = $70-80 \mathrm{~N} / \mathrm{mm}$ measured at the point where the wrist exerted torque on the cuff). Elbow torque was transduced by semiconductor strain gauges mounted on the wrist cuff. The strain gauge bridge circuit was calibrated with a precision spring scale and the circuit's output voltage was linearly related to torque exerted on the cuff between $0-50 \mathrm{Nm}(r=0.95)$.

A visual, compensatory display (CRT), mounted $1 \mathrm{~m}$ in front and approximately $20 \mathrm{~cm}$ higher than eye level, provided information about the required task and subject's ongoing response error. Two horizontal lines were presented on the display: a stationary "reference" (i.e., target) line $3 \mathrm{~mm}$ wide and a movable "tracking" line produced by the electron beam. The width of the reference line required an accuracy of approximately $2.0 \mathrm{Nm}$ for subjects to superimpose the tracking and reference lines. When subjects produced torque on the cuff in the flexion direction, the tracking line moved downward $1 \mathrm{~cm}$ for every $6.7 \mathrm{Nm}$ of torque exerted. Experimenter-induced upward shifts in the tracking line were used both to trigger subjects' responses and to indicate the required torque amplitude (by the separation of the tracking and reference lines). The maximal displacement of the tracking line subtended a visual angle of $3.4^{\circ}$.

\section{Experimental protocols}

Subjects produced sudden changes in steady-state torque (flexion direction) on the isometric wrist cuff in response to an upward displacement of the tracking line. At the experimenter's command, "Ready!", subjects produced a preload of $2.6-3.3 \mathrm{Nm}$ to bring the tracking line slightly below the reference line. A constant preload was used in these experiments to control for the initial level of excitability of the agonist motoneuron pool. Between 1 and $3 \mathrm{sec}$ later, the tracking line was displaced on the display to a new position (rise time $=10 \mathrm{msec}$ ). The vertical excursion of the line represented the amount of torque that the subject was required to produce (in addition to the preload torque). Subjects were instructed to respond to the stimulus by rapidly pulling on the wrist cuff so as to accurately superimpose the tracking line on the reference line and to maintain this position of the tracking line for $1 \mathrm{sec}$. In randomly chosen trials, the tracking line disappeared at the onset of or during the torque response. Subjects were informed of this possibility and were instructed to complete the response as accurately as possible, "as if the tracking line was still present." Subjects were allowed 2-5 practice trials to familiarize themselves with the equipment.

Experiment 1. This experiment was designed to identify different error-reduction mechanisms and the relationship of these mechanisms to visual feedback. Five male and 4 female subjects participated in this experiment. The protocol was composed of 2 sets of 80 trials each. In 1 set of 80 trials, the stimulus amplitude was varied from trial to trial in a pseudorandom order between limits corresponding to required responses of $6.7-40.0 \mathrm{Nm}$. Eight discrete amplitudes were used in the experimental sessions reported below. However, in order to validate a comparison with the other set of 80 trials, 3 subjects were retested with only 3 randomly ordered stimulus amplitudes $(6.7,23.3$, and $40.0 \mathrm{Nm})$, and no differences were found between these 2 sets of experiments. The mean and median stimulus amplitudes in this set of 80 trials were both $23.3 \mathrm{Nm}$. In half of these trials, the tracking line unpredictably disappeared at the onset of subjects' torque responses. When visual feedback was interrupted at the onset of a response, subjects saw the initial upward displacement of the tracking line and, following their reaction time, they saw an additional 10-20 msec of downward displacement caused by their torque response. In the second sct of 80 trials, interruption of visual feedback again occurred unpredictably in half of the trials, but in this group the stimulus amplitude was predictable. Subjects were presented with contiguous blocks of $6.7,23.3$, and $40.0 \mathrm{Nm}$ stimuli (approximately 27 each). Again, mean and median stimulus amplitudes were $23.3 \mathrm{Nm}$. The ordering of the 2 sets of 80 trials was varied among subjects, although differences in ordering produced no distinguishable difference in results.

Experiment 2. This experiment was designed to identify the time following response onset that initial visual detection of torque errors occurs. Eight female and 4 male subjects participated in a second protocol similar to that of the block of 80 trials in experiment 1 with unpredictable stimulus amplitude. This protocol consisted of 200 trials requiring responses between 6.7 and $40.0 \mathrm{Nm}$. Forty different stimulus amplitudes in $0.8 \mathrm{Nm}$ increments were presented. In 140 of these trials, the duration of visual feedback during torque responses was varied from the minimum possible (about $10 \mathrm{msec}$ ) to a maximum of $150 \mathrm{msec}$. In the remaining 60 trials, the tracking line was present throughout the response. For the purposes of data analysis, trials were separated into 6 groups based on the time following response onset at which the tracking line was extinguished: $0-30,31-60,61-90,91-120,121-150 \mathrm{msec}$, and continuous visual feedback. Stimulus amplitudes were equally weighted within each group. The entire 200 trials were presented to the subject in a pseudorandom order.

\section{Data analysis}

The moment of torque response onset was determined by a software algorithm, the accuracy of which was verified by hand measurement $( \pm 5 \mathrm{msec})$. Beginning with response onset, each torque response was arbitrarily subdivided into six $100 \mathrm{msec}$ epochs. Torque was measured at response onset and at the end of each $100 \mathrm{msec}$ epoch. Error was computed at each of these times as the mathematical difference between the required target torque and the actual response torque. Two relationships were determined at the end of each $100 \mathrm{msec}$ epoch: (1) stimulus amplitude (i.e., target torque) versus response torque and (2) response error versus the change in torque over the subsequent $100 \mathrm{msec}$ interval. The former relationship estimated the influence of target torque on the response. The latter relationship estimated the proportion of the response error that was reduced over each $100 \mathrm{msec}$ epoch. The slope of the error versus torque change relationship is referred to as the errorcorrection sensitivity. The analysis of torque responses at $100 \mathrm{msec}$ intervals is limited in its ability to determine precisely the timing of different control mechanisms. However, this technique did provide rough estimates of onset times, which, in some cases, were more precisely determined by analysis of individual trials.

\section{Results}

Three distinct mechanisms controlled torque responses that were made in the presence of visual feedback. The first control mcchanism, termed the initial response, was limited to that portion of the response organized prior to presentation of the triggering stimulus and contributed to the initial rise in torque. The onset of this mechanism was followed at short latency by a corrective adjustment in torque, termed the first adjustment, which was not related to visual detection of torque errors. Approximately 200-250 msec following response onset, another corrective mechanism, termed the second adjustment, produced torque adjustments based on visually detected torque errors.

\section{Initial response}

Subjects' knowledge of the required torque amplitude in advance of stimulus presentation had a marked influence on the initial response strategy. Results from experiment 1 demonstrate this influence, as shown for 1 subject in Figure 1. Representative torque responses are shown for the continuous visual feedback condition when stimulus amplitude was predictable (Fig. 1A) and when it was unpredictable (Fig. $1 B$ ). With predictable stimulus amplitudes, subjects produced a reasonable approximation of the required torque amplitude within the first $100-130 \mathrm{msec}$ (Fig. 1A). In contrast, when the stimulus amplitude was unpredictable (Fig. $1 B$ ), the first $100 \mathrm{msec}$ of response was more variable, falling within an intermediate range of torques. The rise time of torque to this intermediate level was approximately 
Figure 1. Different initial strategies of torque responses depending on stimulus amplitude predictability. Representative samples of torque responses from 1 subject are shown for predictable stimulus amplitudes $(A)$ and for unpredictable stimulus amplitudes $(B)$. Torque $100 \mathrm{msec}$ after response onset is plotted versus stimulus amplitude: in $C$, for predictable stimulus amplitude $(r=0.92, n=40)$ and, in $D$, for unpredictable stimulus amplitude $(r=$ $0.03, n=40$ ). All trials shown had continuous visual feedback. The uneven weighting of stimulus amplitudes in $D$ reflects the distribution of only half of the 80 trial group. Stimulus amplitudes in trials with interrupted visual feedback were equally skewed in the opposite direction (experiment 1).
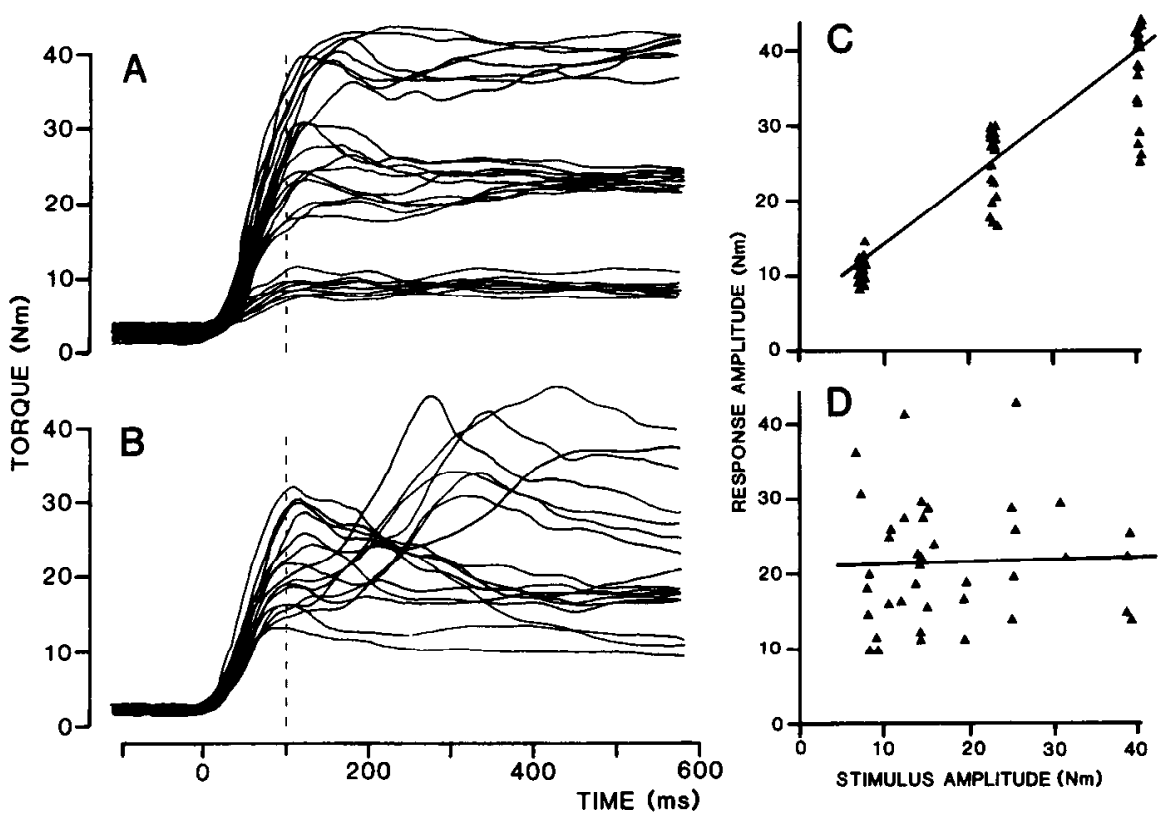

100 msec. With unpredictable targets, however, the rise time of torque to the target level varied considerably from trial to trial because response error varied considerably at $100 \mathrm{msec}$, depending on the actual target torque. Nevertheless, subjects attempted to reach the target as quickly as possible under either stimulus condition. When target torque was unpredictable, the average torque response amplitude at $100 \mathrm{msec}$ was only slightly lower than the mean target torque level $(23.3 \mathrm{Nm})$. For individual subjects, average torque produced at $100 \mathrm{msec}$ ranged from 14.9 to $28.4 \mathrm{Nm}$, with a median value of $20.1 \mathrm{Nm}(n=$ 9). Similar initial response strategies with stimuli having unpredictable characteristics have been reported previously for limb movements (Shae et al., 1981, 1982) and eye movements (Lisberger and Westbrook, 1985).

Mean reaction times with stimuli having unpredictable amplitudes (249 $\pm 65 \mathrm{msec}$, SD; 9 subjects) were slightly longer than those with stimuli having predictable stimulus amplitudes (236 $\pm 71 \mathrm{msec}, \mathrm{SD} ; 9$ subjects). Five of 9 subjects had a significant increase in reaction time $(p \leq 0.05)$ with a mean value of $27 \mathrm{msec}$. This modest increase in reaction time would appear

Table 1. Torque (100 msec) versus stimulus amplitude: unpredictable stimulus amplitude

\begin{tabular}{lll} 
& \multicolumn{2}{l}{ Slope } \\
\cline { 2 - 3 } Subject & No visual feedback & Visual feedback \\
\hline G.L. & 0.05 & 0.07 \\
D.Ro. & $0.14^{a}$ & 0.14 \\
A.M. & 0.01 & 0.09 \\
J.P. & 0.11 & $0.17^{a}$ \\
J.M. & $0.29^{a}$ & $0.33^{a}$ \\
L.C. & 0.06 & 0.03 \\
D.Ru. & $0.23^{a}$ & 0.11 \\
P.C. & $0.20^{a}$ & $0.27^{a}$ \\
B.R. & 0.15 & 0.08 \\
Mean \pm SD & $0.14+0.09$ & $0.14 \pm 0.10$
\end{tabular}

a Significant at $p \leq 0.05$. to be too small to represent a switch from a simple to a choice reaction time response (Hick, 1952).

The rise in torque to intermediate levels, in response to stimuli with unpredictable amplitudes (Fig. $1 B$ ), suggests that target torque information provided by the visual stimulus was not immediately incorporated into this subject's responses. The influence of stimulus amplitude (i.e., target torque) on the torque response was quantitatively demonstrated by plotting the torque produced at specific times, against stimulus amplitude, as shown in Figure $1, C$ and $D$, for 100 msec after response onset. The high slope $(m=0.74)$ and significant correlation $(r=0.92, n=$ 40) of these variables when stimulus amplitude was predictable (Fig. 1C) may simply reflect the subject's certainty prior to stimulus presentation of how much torque would be required. In contrast, stimulus amplitude had no significant influence on this subject's response at $100 \mathrm{msec}(r=0.03, n=40)$ for trials with unpredictable stimulus amplitude (Fig. $1 D$ ). For trials with unpredictable stimulus amplitude and continuous visual feedback, stimulus amplitude was significantly correlated with torque at $100 \mathrm{msec}$ in only 3 of the 9 subjects (Table 1 , right), but the mean slope of this relationship for all nine subjects was modest $(m=0.14)$. This relationship was similar for trials without visual feedback (Table 1, left), indicating that the presence or absence of visual feedback has no effect on this relationship. Thus, it appears that stimulus amplitude began to influence response torque by $100 \mathrm{msec}$ after response onset in some subjects; however, in most subjects this influence was delayed until after 100 msec.

\section{First adjustment}

Following the initial response to unpredictably sized stimuli, torque trajectories diverged from the intermediate range with a sequence of corrective adjustments (Fig. $1 B$ ). Using the methods described below, torque changes between 100 and $200 \mathrm{msec}$ in responses to predictably sized stimuli (Fig. $1 A$ ) were also determined to reduce response error. However, the timing and mechanisms underlying error corrections were more clearly demonstrated for responses to unpredictably sized stimuli be- 

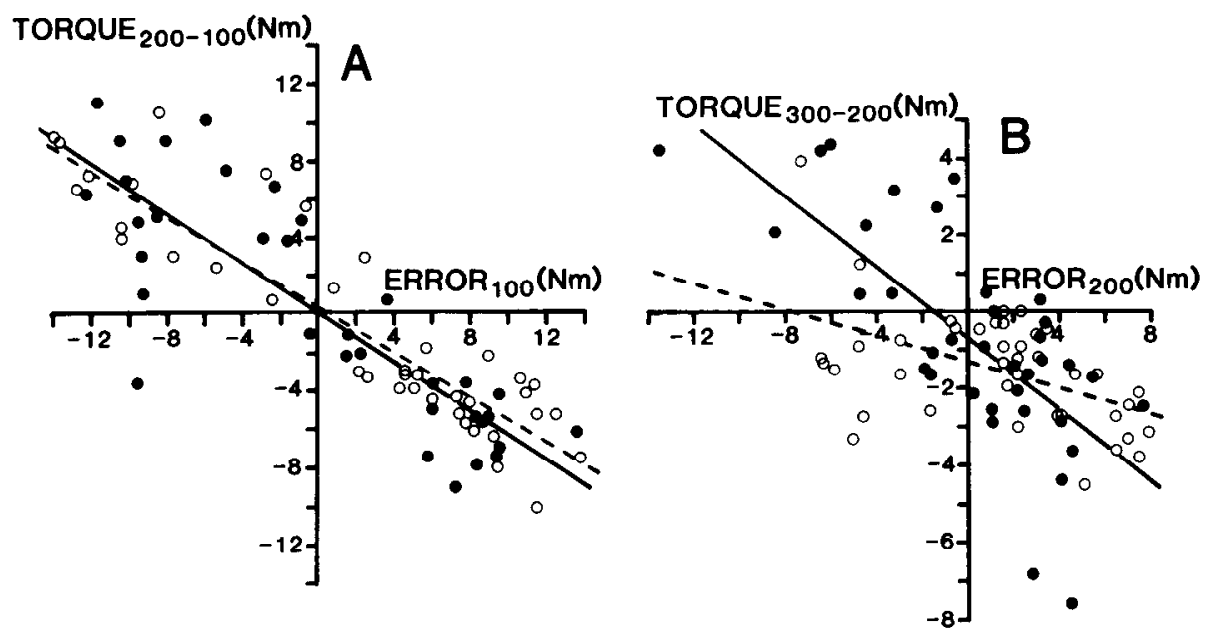

Figure 2. Error correction is demonstrated by a significant correlation between error and subsequent torque change over $100 \mathrm{msec}$ intervals for 1 subject. Plots are for the unpredictable stimulus amplitude condition. $A$, Error at $100 \mathrm{msec}$ plotted against the torque change between 100 and 200 msec: filled symbols and solid line for continuous visual feedback $(m=-0.65, r=-0.86)$ and open symbols and dashed line for interrupted visual feedback $(m=-0.59$, $r=-0.92) . B$, Error at $200 \mathrm{msec}$ and the torque change between 200 and 300 msec: filled symbols and solid line for continuous visual feedback $(m=-0.48$, $r=-0.73$ ) and open symbols and dashed line for interrupted visual feedback $(m=-0.18, r=-0.50)$ (experiment 1). cause initial errors were enhanced by greater stimulus uncertainty; consequently, responses to unpredictably sized stimuli will be the focus of subsequent analyses.

The second 100 msec epoch contained a substantial reduction in initial response error and, in all subjects, was accompanied by the development of a highly significant correlation between stimulus ampitude and response torque. This error reduction, termed the first adjustment, occurred independently of visual feedback, as indicated by the relationship between response error at $100 \mathrm{msec}$ and the subsequent change in torque during the interval 100-200 msec. The slope of this relationship represents the proportion of error that was eliminated in a $100 \mathrm{msec}$ period, termed the error correction sensitivity. In Figure $2 A$, error at $100 \mathrm{msec}$ was plotted against the subsequent torque change between 100-200 msec for trials where stimulus amplitude was unpredictable (one subject). The filled symbols are from trials with continuous visual feedback, and the open symbols are from trials where the visual display was blanked at the beginning of the subject's response. The straight lines are from a least-squares regression, and their slopes are $m=-0.65$ (solid line, visual feedback) and $m=-0.59$ (dashed line, no visual feedback). Because a slope of $m=-1.00$ would indicate the complete elimination of error, these slopes indicate that slightly over half the error existing at the end of the first $100 \mathrm{msec}$ of responses was corrected during the second 100 msec. Results from the other 8 subjects were comparable (Table 2). Average slope values with visual feedback were not significantly different from those with no visual feedback ( $t$ test, $p>0.05$ ), indicating that the error-correction sensitivity of the first adjustment mechanism is independent of visual feedback. Therefore, the onset of control based on visual detection of a torque error must occur later than the 100-200 msec epoch.

\section{Second adjustment}

Changes in torque between 200 and $300 \mathrm{msec}$ further reduced response errors, but the amount of error reduction was small or insignificant when visual feedback was interrupted. This influence of visual feedback was demonstrated by comparing the error $200 \mathrm{msec}$ after response onset with the subsequent change in torque between 200 and $300 \mathrm{msec}$. In Figure $2 B$, the solid line and filled symbols demonstrate this relationship for trials with visual feedback and the dashed line and open symbols for trials with interrupted visual feedback (same subject as Fig. 2A).
When visual feedback was continuously provided, the slope was $m=-0.48$, whereas when visual feedback was interrupted, it was only $m=-0.18$. In agreement with 2 previous studies (Keele and Posner, 1968; Beggs and Howarth, 1970), these results indicate that the onset of visually mediated error correction occurs during the 200-300 msec epoch.

Analysis of the data from all 9 subjects in experiment 1 supports this conclusion (Table 3 ). The average error-correction sensitivity of all active mechanisms during the $200-300 \mathrm{msec}$ interval without visual feedback $(m=0.22)$ was about half that with visual feedback ( $m=0.47)$, and the 2 means were significantly different ( $t$ test, $p \leq 0.05$ ). The residual error reduction that occurs between $200-300 \mathrm{msec}$ in the absence of visual feedback may be mediated by the same mechanism that reduced error during the 100-200 msec epoch (first adjustment).

The analysis used in Figure 2 distinguishes the polarity of error as positive (overshoot) or negative (undershoot). Estimates of response accuracy are more commonly determined with the absolute value of error (sometimes referred to as "modulus mean error"), which does not distinguish between overshooting and undershooting (Poulton, 1981). The time at which visual feedback begins to modify torque trajectories can also be demonstrated with measurements of response accuracy.

The average absolute value of error ( 9 subjects) is plotted Table 2. Torque (200-100 msec) versus error (100 msec):
unpredictable stimulus amplitude

\begin{tabular}{lll} 
& \multicolumn{2}{l}{ Slope } \\
\cline { 2 - 3 } Subject & No visual feedback & Visual feedback \\
\hline G.L. & -0.33 & -0.29 \\
D.Ro. & -0.30 & -0.39 \\
A.M. & -0.22 & -0.30 \\
J.P. & -0.34 & -0.50 \\
J.M. & -0.66 & -0.59 \\
L.C. & -0.25 & -0.21 \\
D.Ru. & -0.21 & -0.26 \\
P.C. & -0.59 & -0.65 \\
B.R. & -0.40 & -0.43 \\
\multicolumn{1}{c}{ Mean + SD } & $-0.36 \pm 0.16$ & $-0.40 \pm 0.15$ \\
\hline
\end{tabular}

All data were significant at $p \leq 0.05$. 


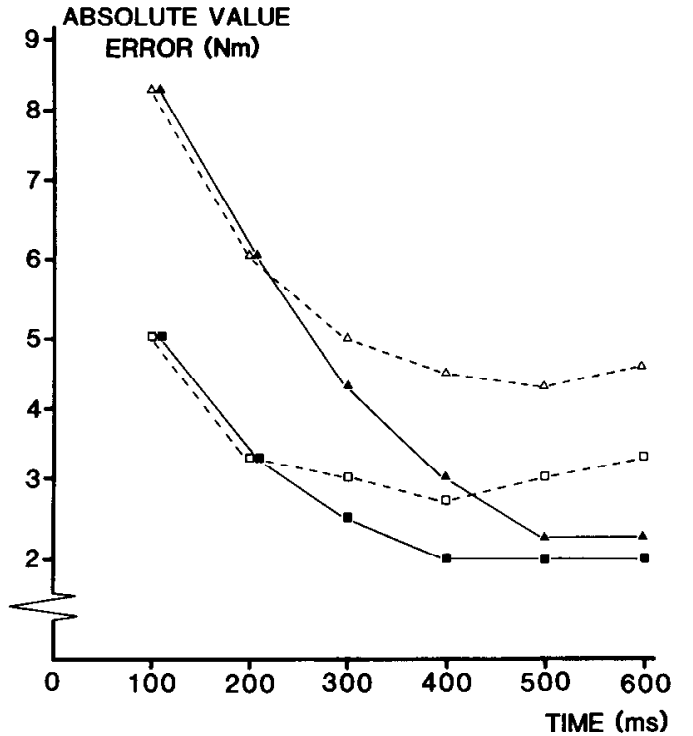

Figure 3. Error at $100 \mathrm{msec}$ depends on stimulus amplitude predictability and, at $600 \mathrm{msec}$, principally on the availability of visual feedback. Absolute value of error is plotted at $100 \mathrm{msec}$ intervals and each point represents the average error for 9 subjects. Filled symbols and solid lines represent trials with continuous visual fccdback, and open symbols and dashed lines represent trials with interrupted feedback. Triangles represent trials with unpredictable stimulus amplitude, and squares represent trials with predictable stimulus amplitude (experiment 1).

against the time after response onset in Figure 3 for the 4 stimulus and visual feedback combinations: predictable or unpredictable stimulus amplitude and continuous or interrupted visual feedback. When stimulus amplitude was unpredictable (triangles), the mean absolute value of the error $100 \mathrm{msec}$ after response onset was $8.3 \mathrm{Nm}$. This is precisely the value expected if assuming intermediate sized initial responses of $23.3 \mathrm{Nm}$ because of the even weighting of stimulus amplitudes on either side of the mean: The average error should equal one-quarter of the total torque range of stimuli $(6.7-40.0 \mathrm{Nm})$. When stimulus amplitude was predictable (squares), the average error at
Table 3. Torque (300-200 msec) versus error ( $200 \mathrm{msec})$ : unpredictable stimulus amplitude

\begin{tabular}{lll} 
& \multicolumn{2}{l}{ Slope } \\
\cline { 2 - 3 } Subject & No visual feedback & Visual feedback \\
\hline G.L. & $-0.30^{a}$ & $-0.37^{a}$ \\
D.Ro. & $-0.32^{a}$ & $-0.49^{a}$ \\
A.M. & $-0.14^{a}$ & $-0.24^{a}$ \\
J.P. & $-0.28^{a}$ & $-0.52^{a}$ \\
J.M. & -0.08 & $-0.65^{a}$ \\
L.C. & $-0.21^{a}$ & $-0.42^{a}$ \\
D.Ru. & $-0.13^{a}$ & $-0.29^{a}$ \\
P.C. & $-0.18^{a}$ & $-0.48^{a}$ \\
B.R. & $-0.31^{a}$ & $-0.73^{a}$ \\
\multicolumn{1}{c}{ Mean \pm SD } & $-0.22 \pm 0.09$ & $-0.47 \pm 0.16$ \\
\hline
\end{tabular}

${ }^{a}$ Significant at $p \leq 0.05$.

$100 \mathrm{msec}$ was approximatcly $5 \mathrm{Nm}$. (The difference in accuracy at $100 \mathrm{msec}$ resulting from stimulus predictability was reflected by the distinctive initial response strategies illustrated in Fig. 1.)

During the interval 100-200 msec after response onset, error declined as a result of the first adjustment, as previously demonstrated in Figure 2A. The close correspondence of plots in Figure 3 for visual feedback and no visual feedback conditions indicates that visual feedback had no effect on the rate of error reduction during this $100 \mathrm{msec}$ period. However, during the subsequent $100 \mathrm{msec}$ interval (200-300 msec), each pair of plots diverges. Error continued to decline rapidly when visual feedback was provided, whereas it began to stabilize when visual feedback was interrupted. The divergence of each pair of plots demonstrates the effect of visual feedback. The decline of average error after $200 \mathrm{msec}$ when visual feedback was interrupted, although small, may reflect a persistence of the first adjustment mechanism. Plots with similar visual feedback conditions converge $500-600 \mathrm{msec}$ after response onset, indicating that steadystate accuracy is principally determined by visual feedback conditions rather than the initial predictability of the target location.

\begin{abstract}
Figure 4. Visually mediated error corrections. In $A$, heavy traces are average torque responses with continuous visual feedback and thin traces are averages from trials with interrupted visual feedback. The upper pair of torque records is from trials in which positive errors were greater than $10 \mathrm{Nm}$ at 100 $\mathrm{msec}$ and the lower pair from trials with negative errors greater than $-10 \mathrm{Nm}$ at $100 \mathrm{mscc}$. In $B$, the onset of visually mediated torque corrections in individual trials is identified with arrows. Trials in $B$ are from the lower average in $A$ with a heavy line. Torque calibration applies to both averages and single trial records from this subject (experiment 1).
\end{abstract}

A

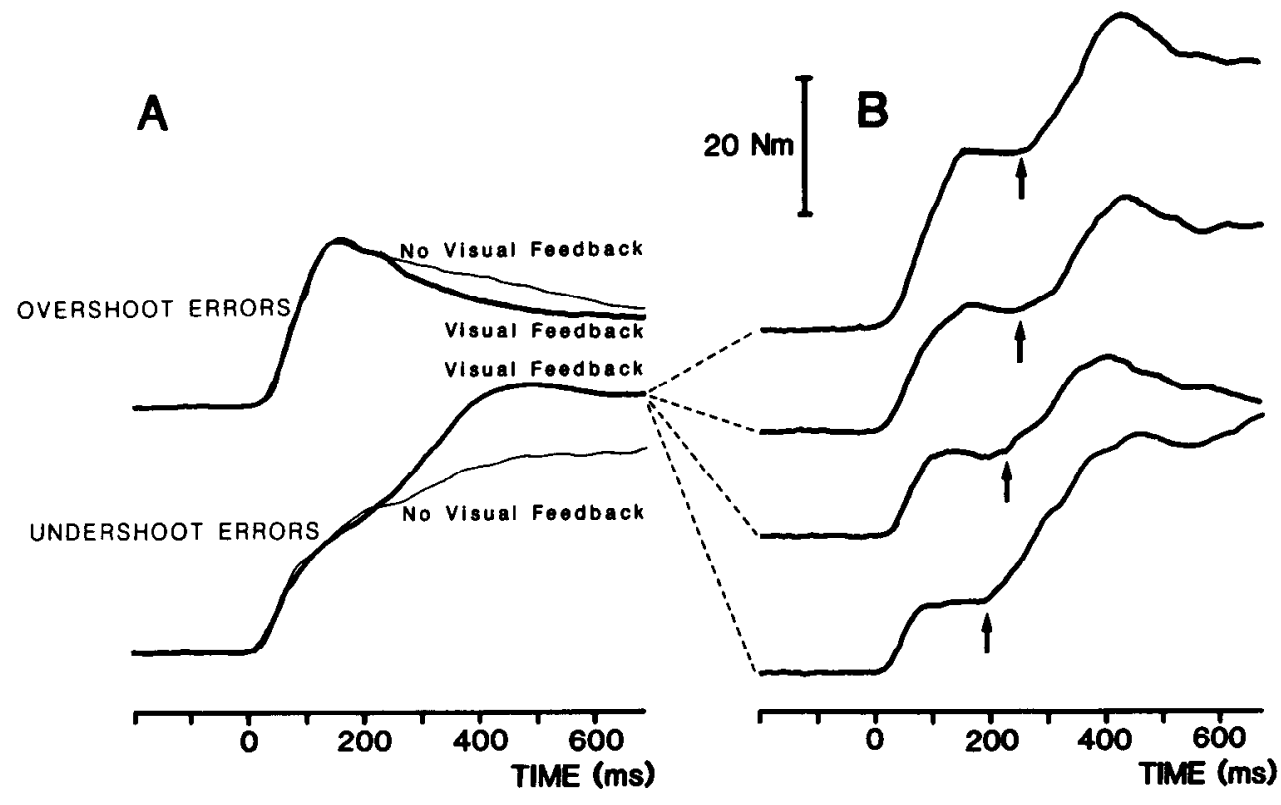




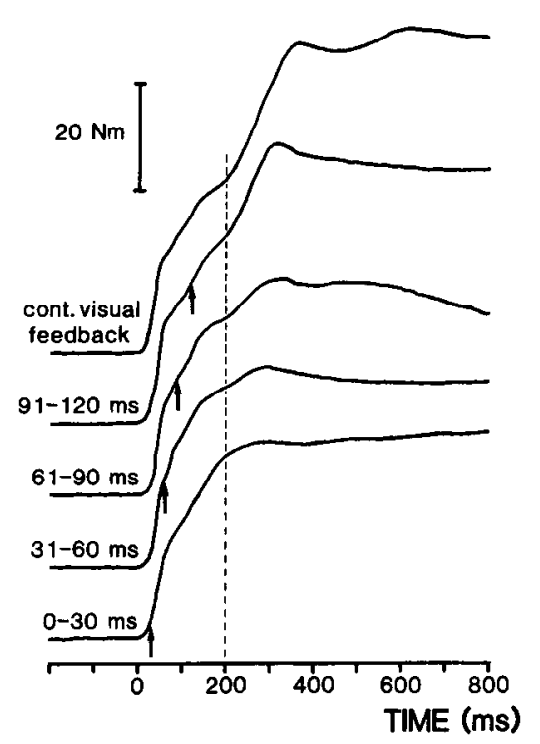

Figure 5. Torque response averages from trials with different durations of visual feedback from 1 subject. Each trace represents the average of torque responses that undershot the target by at lesat $10 \mathrm{Nm}$ at 100 msec. The time intervals on the left represent the range of timing following response onset at which the visual display was blanked. Arrows indicate the latest time that visual feedback was provided for any trial in an average (experiment 2 ).

When visual feedback was interrupted, some subjects' error increased after $400-500 \mathrm{msec}$, as reflected in the averages of Figure 3. This increase in error resulted from a gradual decline in the amount of torque being generated in the absence of visual feedback (undershooting the target), perhaps as a result of "sag" of fast twitch motor units (Burke et al., 1973) or motoneuronal accommodative processes during the application of constant effort (Kernell, 1975).

\section{Examples of discrete visually mediated torque adjustments}

The presence of discrete, visually mediated error corrections was revealed by averaging selected torque responses. From the 80 trials in each experiment with unpredictably sized stimuli, only those with errors greater than $10 \mathrm{Nm}$ at $100 \mathrm{msec}$ were selected. These trials were broken down into 4 groups based on whether the torque error at $100 \mathrm{msec}$ was positive or negative and whether or not visual feedback was interrupted at response onset. An average torque response was calculated for each of these groups, as shown in Figure $4 A$ for a typical subject. Comparison of the averages for trials with large positive errors (Fig. $4 A$, top) reveals a slightly faster decline in torque when visual feedback was provided (heavy trace, $n=10$ ) compared to when it was interrupted (thin trace, $n=7$ ). A more distinct difference existed for the average torque responses in trials with large negative errors (Fig. $4 A$, bottom). When visual feedback was provided, a sharp increase in torque began about $200 \mathrm{msec}$ after response onset (heavy trace, $n=9$ ), whereas only a modest torque change was observed when visual feedback was interrupted (thin trace, $n=11$ ).

An estimate of the delay from the beginning of torque responses to the onset of visually guided corrections can be obtained from selected trials. The 4 single trials shown in Figure $4 B$ are components of the average response shown in Figure $4 A$ (lower, heavy line). In each of these individual responses, a distinct increase in torque occurred at around $200 \mathrm{msec}$ after

\begin{tabular}{|c|c|}
\hline Subject & $\underset{(\mathrm{msec})}{\operatorname{Mean}} \pm \mathrm{SD}$ \\
\hline G.L. & $248 \pm 29(n=6)$ \\
\hline D.Ro. & $251 \pm 18(n=5)$ \\
\hline A.M. & $234 \pm 17(n=2)$ \\
\hline J.P. & $251 \pm 16(n=5)$ \\
\hline J.M. & $224 \pm 9 \quad(n=7)$ \\
\hline L.C. & $204 \pm 31(n=9)$ \\
\hline D.Ru. & $257 \pm 24(n=5)$ \\
\hline P.C. & $221 \pm 18(n=5)$ \\
\hline B.R. & $204 \pm 18(n=14)$ \\
\hline Mean $\pm \mathrm{SD}$ & $233 \pm 20(n=9$ subjects $)$ \\
\hline
\end{tabular}

response onset (arrows). Distinct increases in torque were never seen this late in individual responses produced without visual feedback. Therefore, these discrete inflections in torque trajectories are thought to be visually mediated error corrections.

In the 9 subjects tested, the number of large-amplitude, positively directed error corrections varied from 2 to 14 per 40 trials with visual feedback. The time from response onset to the onset of the presumed visual correction was estimated for each of these selected torque responses. Average results from all 9 subjects are listed in Table 4 . The mean latency of visual corrections varied considerably among subjects (204-257 msec) and the average for all subjects was $233 \pm 20 \mathrm{msec}$ (SD). Individual latency measurements also varied considerably for single subjects, as indicated by the SD in Table 4 . The earliest recorded, visually mediated correction was $160 \mathrm{msec}$ in subject L.C.

\section{Visual feedback loop delays}

The visual feedback processing time can be conceptually divided into 2 epochs: the time for response onset to the visual detection of a torque error followed by the delay for the neuromuscular system to process this information and modify torque. A variation of experiment 1 was performed to ascertain the durations of these 2 epochs. As described in Materials and Methods, experiment 2 consisted entirely of trials in which stimulus amplitude was unpredictable. Visual feedback was interrupted during most trials, but tracking line extinction was varied unpredictably between response onset and $150 \mathrm{msec}$ later. This procedure was used to determine the minimum duration of visual feedback required to produce the visually mediated error corrections observed at about $200 \mathrm{msec}$.

Torque responses with large negative errors (greater than 10 $\mathrm{Nm}$ ) at $100 \mathrm{msec}$ were selected from these experiments. These responses were broken down into groups based on the duration of visual feedback provided, beginning with $30 \mathrm{msec}$ of feedback or less and increasing up to $150 \mathrm{msec}$ in $30 \mathrm{msec}$ increments. A control group was also included in which visual feedback was continuously provided. Each group of trials was averaged as shown for a representative subject in Figure 5. Each torque trace is an average of 10-15 trials, and the arrows indicate the latest time that visual feedback was provided (i.e., when it was interrupted) for any trial in a given average. The vertical, dashed line marks the $200 \mathrm{msec}$ point and indicates the expected time of visually mediated corrections. In this example, clear evidence 


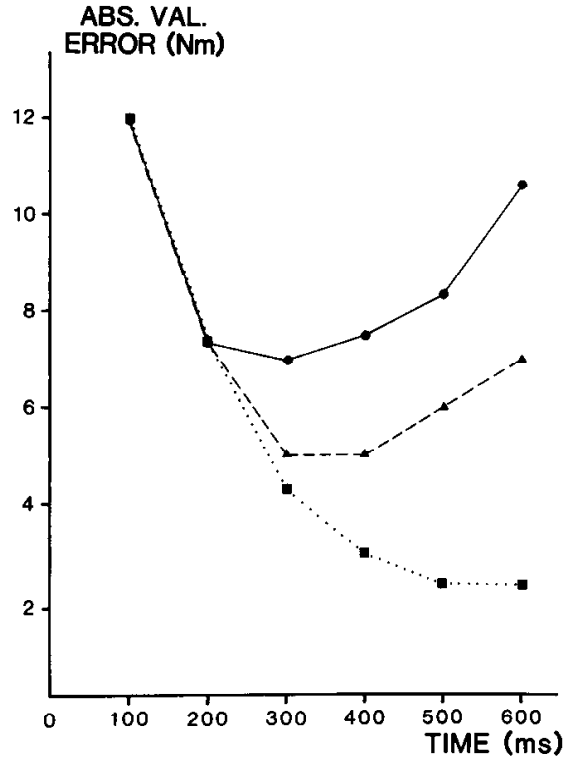

Figure 6. Plot of the absolute value of error versus time following response onset for 1 subject. Symbols represent the average error for trials with visual feedback interrupted between $0-30 \mathrm{msec}$ following response onset (circles), visual feedback interrupted between $61-90 \mathrm{msec}$ (triangles), and continuous visual feedback (squares). Error values for each plot have been normalized with respect to error at $100 \mathrm{msec}(\mathrm{ex}$ periment 2).

of a visual correction appeared in the average from trials with 61-90 msec of visual feedback and correction amplitude increased up to near maximum in the $91-120 \mathrm{msec}$ average. In similar averages, all subjects showed some error correction in the $61-90 \mathrm{msec}$ group. These results suggest that the initial visual detection of torque errors can occur between about 60 $120 \mathrm{msec}$. The difference between this range and the mean delay to the onset of error correction (i.e., $233 \mathrm{msec}$; Table 4) provides a rough estimate of the delay from error detection to correction: 110-170 msec. The low end of this range corresponds closely to the fastest estimates of Smith and Bowen (1980) and Carlton (1981).

\section{Relationship of the first and second adjustments}

The combination of a 110-170 msec delay between visual error detection and correction and an intervening first adjustment creates a potentially unstable condition. The torque error that existed at the onset of the second adjustment was no longer the same as that visually detected $110-170 \mathrm{msec}$ earlier, at the onset of the first adjustment. The polarity of the error may even reverse between 100 and $200 \mathrm{msec}$ (e.g., undershoot to overshoot) as the result of an overcompensated first adjustment. Such a reversal of polarity would cause an inappropriatcly directed second adjustment if the second adjustment was performed independently of the first adjustment.

One test for an interdependence of first and second adjustments is to determine if appropriately directed second adjustments are produced following overcompensated first adjustments. For this purpose, all trials involving unpredictable stimulus amplitudes and continuous visual feedback were separated into 2 groups based on whether or not the polarity of error changed as a result of the first adjustment (experiments 1 and 2). The group of trials in which polarity changed between
Table 5. Proportion of appropriately directed second adjustments all trials except error at $100 \mathrm{msec}<2.0 \mathrm{Nm}$

\begin{tabular}{lcc} 
& \multicolumn{2}{c}{ Polarity } \\
\cline { 2 - 3 } Subject & Unchanged & Changed \\
\hline A.F. & $54 / 56$ & $1 / 1$ \\
A.M. & $37 / 40$ & $0 / 0$ \\
S.W. & $28 / 32$ & $7 / 8$ \\
D.T. & $37 / 46$ & $6 / 6$ \\
C.C. & $40 / 46$ & $3 / 3$ \\
M.F. & $45 / 46$ & $2 / 2$ \\
L.H. & $52 / 59$ & $4 / 4$ \\
Y.F. & $59 / 60$ & $1 / 1$ \\
C.M. & $17 / 21$ & $3 / 4$ \\
P.C. & $18 / 20$ & $1 / 2$ \\
M.M. & $21 / 32$ & $0 / 3$ \\
R.F. & $53 / 55$ & $2 / 3$ \\
A.M. & $25 / 30$ & $0 / 1$ \\
P.C. & $19 / 19$ & $0 / 1$ \\
D.Ru. & $19 / 25$ & $1 / 5$ \\
G.L. & $35 / 37$ & $1 / 2$ \\
J.M. & $16 / 17$ & $0 / 3$ \\
L.C. & $25 / 28$ & $1 / 1$ \\
D.Ro. & $22 / 25$ & $4 / 9$ \\
C.M. & $19 / 20$ & $4 / 4$ \\
J.P. & $19 / 21$ & $6 / 7$ \\
Total & $660 / 735$ & $47 / 70$ \\
Percent appropriate & 90 & 67 \\
\hline & & \\
\hline
\end{tabular}

100 and $200 \mathrm{msec}$ was generally quite small because, on the average, the first adjustment corrected for only about half the preceding error during this interval (Fig. $2 A$ ). The second adjustment in each of these reversal-of-error trials was evaluated to determine whether its direction was appropriate. If the first and second adjustments were produced by completely independent mechanisms, changes in error polarity between 100 and $200 \mathrm{msec}$ should have resulted in inappropriately directed second adjustments.

Table 5 summarizes these results in the form of a ratio: the number of appropriately directed second adjustments to the total number of trials in a given group. Two ratios are listed for each subject, one for trials in which the polarity of error reversed between 100 and $200 \mathrm{msec}$ and the other in which it did not reverse. Only those trials were used in which the error existing at $100 \mathrm{msec}$ exceeded the minimum, average steady-state error (approximately $2.0 \mathrm{Nm}$ at $600 \mathrm{msec}$, Fig. 3). The totals at the bottom of Table 5 indicate that, when the polarity of error changed between 100 and $200 \mathrm{msec}, 67 \%$ of the subsequent second adjustments were appropriately directed compared to $90 \%$ for trials with no change in error polarity. The $10 \%$ of inappropriately directed second adjustments, when error polarity did not reverse during the preceding $100 \mathrm{msec}$, is presumed to represent the inherent noise level of the corrective mechanism. The data in Table 5 argue against complete independence of the first and second adjustments, a condition that should have resulted in only $10 \%$ of second adjustments, subsequent to error polarity reversal, being appropriately directed instead of $67 \%$. 


\section{Time course of visuomotor control}

The visually mediated correction data presented thus far have been primarily concerned with the first visually controlled 100 msec epoch: 200-300 msec after response onset. A complete description of the visually mediated error-correcting system must also include longer-term error correction and its degree of dependence on the continuity of visual feedback.

Error correction at longer latencies was found to depend upon the persistence of visual feedback. In Figure 6, results from 1 subject demonstrate the time course of average absolute value of error for 3 trial groups from experiment 2: $0-30 \mathrm{msec}$ of visual feedback (solid line and circles), $61-90 \mathrm{msec}$ of visual feedback (dashed line and triangles), and continuous visual feedback (dotted line and squares). Because the average error at 100 msec was not exactly the same for each small group of trials $(n=10-15)$, these plots were normalized with respect to error at $110 \mathrm{msec}$. For trials with minimal visual feedback (circles), a relatively small reduction in error occurred after the $200 \mathrm{msec}$. When 61-90 msec of visual feedback was provided (triangles), error reduction persisted through $300 \mathrm{msec}$ (second adjustment). Finally, when visual feedback was continuously available (squares), visually mediated error reduction occurred over a period up to $500 \mathrm{msec}$, until steady-state accuracy was reached. These data demonstrate a direct coupling between the visual error signal and long-term error correction.

Since average error does not decline after $500 \mathrm{msec}$, it is not obvious whether error correction continues after this point and, if so, whether it is visually mediated. The net error correction sensitivity of all active mechanisms (slopes in Fig. 2) provides a more sensitive test for the action of the visual correction mechanism. Figure 7 charts the time course of error correction sensitivity over the first $600 \mathrm{msec}$ of torque responses with unpredictable stimulus amplitudes. Each point represents the mean error correction sensitivity during the inclusive $100 \mathrm{msec}$ epoch ( 9 subjects, experiment 1$)$. The dashed line and open triangles represent the interrupted visual feedback condition and the filled triangles and thin solid line represent the continuous visual feedback condition. The mathematical difference between these 2 plots (circles and heavy line) represents the effect of visual feedback on error correction. It is apparent that the visual feedback mechanism becomes active at around $200 \mathrm{msec}$ with a relatively constant error correction sensitivity of about onequarter up through $600 \mathrm{msec}$. These results suggest that the visual system is responsible for the accurate maintenance of steady-state torque.

\section{Discussion}

When visual feedback was provided, accurate changes in torque were achieved through the sequential recruitment of at least 3 control mechanisms, termed the initial response, first adjustment, and second adjustment. The term initial response refers to that component of the triggered motor command that was organized prior to the presentation of the stimulus and contributed to the initial rise in torque. The initial response was followed at short latency by corrective modification of torque levels. Corrective modification was shown to be controlled by 2 distinct mechanisms, the first of which was independent of visual feedback (first adjustment). The second corrective mechanism, which became active approximately $200 \mathrm{msec}$ after response onset, was dependent on visual feedback (second adjustment). The decomposition of torque responses into these constituent com-

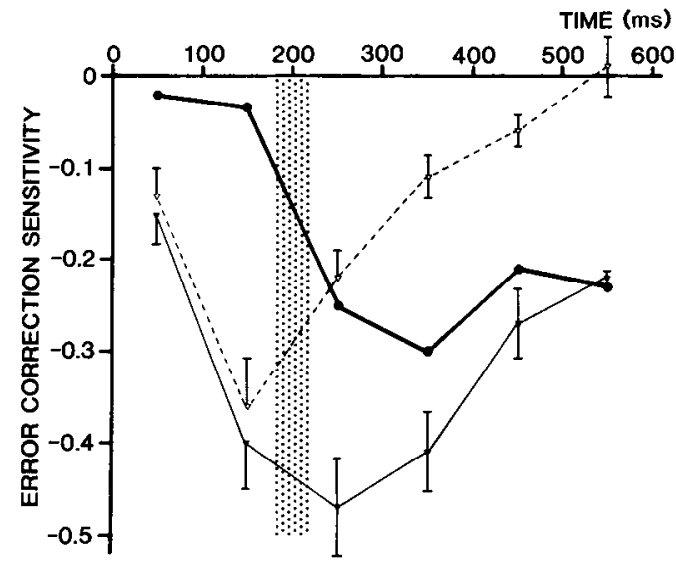

Figure 7. Error correction sensitivity (i.e., slope of change in torque versus error relationship) over time. Each point represents mean slope $( \pm \mathrm{SE})$ for 9 subjects. Filled triangles and thin solid line for continuous visual feedback, open triangles and thin dashed line for no visual feedback, and filled circles and heavy line for differences. Stippling represents the approximate onset time of visual feedback control (experiment 1).

ponents and the characterization of each was the goal of this study.

\section{Initial response and first adjustment}

The visual stimulus that triggered torque responses contained information of both temporal and metrical nature. The temporal information (tracking line movement) cued subjects to initiate their responses as quickly thereafter as possible. Metrical information was provided by the distance of tracking line displacement (relative to the reference line) and informed subjects of the required response amplitude. When stimulus amplitude was predictable, the metrical information contained in the stimulus was redundant and subjects' torque at $100 \mathrm{msec}$ was highly correlated with stimulus amplitude (Fig. $1 C$ ). In contrast, when stimulus amplitude was unpredictable, torque at $100 \mathrm{msec}$ was only weakly related to stimulus amplitude (Fig. 1D). At face value, the results from the subject in Figure 1 indicate that the initial response was organized prior to stimulus presentation and that prior certainty of the required torque amplitude was reflected by the accuracy of the response at $100 \mathrm{msec}$.

However, a comparison of all 9 subjects suggested that responses were sometimes updated during the initial $100 \mathrm{msec}$ epoch. The relationship between the unpredictable stimulus amplitude and the torque at $100 \mathrm{msec}$ had a positive slope for all subjects and was significantly correlated $(p \leq 0.05)$ for almost half (Table 1). This result demonstrates the incorporation of stimulus amplitude information into the response prior to 100 msec. However, the absence or weak effect of stimulus amplitude on response torque at $100 \mathrm{msec}$ in some subjects indicates that temporal and metrical components of the stimulus are processed separately (Megaw, 1974; Ghez et al., 1983; Favilla et al., 1985). The simplest explanation for the variability of this effect among subjects is that the first adjustment mechanism becomes active at different times for different subjects. In some subjects, updating of the initial response by the first adjustment commences during the first $100 \mathrm{msec}$, whereas in others updating by the first adjustment is delayed until the second 100 msec.

It is possible that metrical information contained in the visual stimulus is processed by the motor system similarly to its later 
treatment of visually detected torque errors. The separation of the tracking and reference lines produced by the trigger stimulus might have been interpreted as an initial form of error, albeit experimenter-induced rather than response-induced. As a result of a prolonged processing time, this metrical information was incorporated into the response as an upward or downward adjustment in torque (first adjustment) some time after response onset. This required that the stimulus amplitude information be compared to some representation of the initial response in order to determine the status of torque production prior to the onset of the first adjustment.

Three possible representations of the intial response are prediction, kinesthetic feedback, and corollary discharge. With unpredictable stimulus amplitudes, a predictive mechanism could presume that the initial response will have attained a particular (intermediate) torque level at the beginning of the first adjustment. However, the large variability in torque at $100 \mathrm{msec}$ (Fig. $1 D$ ) makes this possibility unlikely since any consistent prediction of torque would be highly inaccurate. Kinesthetic input has been shown to produce instructionally dependent EMG responses within $50 \mathrm{msec}$ of limb perturbations in certain kinds of position-holding tasks (Hammond, 1960; Evarts and Tanji, 1974). Therefore, kinesthetic input from the limb might provide the required representation of motor output during the initial response. However, a number of studies testing for the presence of a kinesthetic error-correcting mechanism have mostly produced negative results (Polit and Bizzi, 1979; Kelso et al., 1980). By exclusion, a corollary discharge mechanism seems the most likely source of the initial response representation required for the organization of the first adjustment. Evidence exists for the action of corollary discharge in voluntary limb movements (McCloskey, 1981; Conke and Diggles, 1984). Furthermore, the interdependence of the first and second adjustments demonstrated in Table 5 can also be explained by the action of a corollary discharge mechanism. The confirmation of this possibility, however, awaits a more direct experimental test.

\section{Second adjustment}

The measurements of visuomotor control timing made in these experiments largely confirmed previous work from other laboratories. The average delay from response onset to the beginning of visually mediated corrective action $(200-250 \mathrm{msec}$, in agreement with Keele and Posner, 1968) was based on the identification of discrete error corrections in individual torque records. The interval from visual error detection to error correction was estimated at 110-170 msec, in agreement with Smith and Bowen (1980) and Carlton (1981). Visually mediated control was shown to span the interval of $200-600 \mathrm{msec}$ (Fig. 7) with no evidence of a decline in error-correction sensitivity. Therefore, it is likely that visual input is useful for the accurate maintenance of steady-state torque in these motor tasks.

While previous studies have characterized some of the temporal properties of visual feedback control, the present experiments have also revealed some metrical characteristics of this corrective system. Error correction sensitivity of torque adjustments was assessed over $100 \mathrm{msec}$ epochs by plotting error against subsequent modifications in torque (Fig. 2). The slope of this relationship indicated that the preexisting error was reduced by a factor of almost one-half during the first $100 \mathrm{msec}$ of visual control (Table 2). However, all active error-correction mechanisms contributed additively to this estimate of sensitiv- ity. The visual error-correction mechanism was shown to reduce overall error by about one-quarter over each $100 \mathrm{msec}$ epoch that this mechanism was active (Fig. 7).

\section{Control system stability}

Previous studies of rapid, goal-directed limb movements have addressed the need to compensate for mechanical instability of the limb. For example, both the antagonist burst (Meinck et al., 1984) and the second agonist burst (Wadman et al., 1979; Ghez and Martin, 1982) of the well-known 3-burst pattern (Wachholder and Alterburger, 1926; Hallett et al., 1975) have been assigned the role of viscous damping. Negative feedback systems can have other potential sources of instability, notably high gain and long loop time; a careful balancing of these 2 variables is required in the design of a servomechanism. The net sensitivity of all error-correction mechanisms appears to have been held well below unity in order to prevent the reversal of error polarity over a $100 \mathrm{msec}$ interval, the approximate loop time of the visual correction mechanism. Although occasional overcorrection for errors was observed, information transfer from the first to the second adjustments appeared to compensate for and thus prevent oscillation (Table 5). One mechanism that might be responsible for the interdependence of adjacent torque adjustments is the comparison of corollary discharge with reafferent input (McCloskey, 1981; Cooke and Diggles, 1984). Therefore, potentially unstable behavior due to a long loop delay appears to be prevented by low feedback gain and some form of information transfer from one control mechanism to the next.

Corrections involving increased torque (undershoot errors) were more distinct and had a faster time course than those requiring decreased torque. This result may be related to the muscular activation strategy used by subjects to produce isometric torque: The antagonist muscle (triceps) was only weakly activated. Consequently, corrections for overshooting the target were produced by decreasing agonist muscle activity. The differences in shape of overshoot and undershoot corrections might then be explained by parallel differences in the rise and relaxation times of a muscle twitch (Milner-Brown et al., 1973).

\section{Incorporation of target torque into motor commands}

During accurate changes in joint torque, the final limb torque was gradually achieved through a series of 3 approximations. Each approximation was produced by a relatively distinct control mechanism based on information about the anticipated target torque, the actual target torque, and the ongoing torque response. The use of the successive approximation strategy allowed subjects to initiate torque responses as quickly as possible after the visual stimulus. Subjects controlled their torque responses with these same 3 mechanisms, independently of target predictability.

The successive approximation strategy for guiding joint torque to a target differs in some respects from previously hypothesized strategies of limb control. During the initial response, torque response variability depended on the mean torque amplitude (when target torque was predictable), as well as the predictability of target torque (Fig. 1). Larger torque responses were more variable (Fig. $1 C$ ), and decreased predictability was associated with increased torque variability (Fig. 1D). The "impulse variability" model of Schmidt et al. (1979) accounts for variability due to mean torque amplitude but not for variability due to target predictability. During the first adjustment, torque re- 
sponses were updated independently of visual feedback (Fig. 2). Schmidt et al. (1979), Keele (1968), and Polit and Bizzi (1979) suggested that targeted movements can be executed without sensory feedback and, by implication, without response updating. However, as demonstrated in the present study and by Ghez et al. (1983) and Favilla et al. (1985), the target itself is used to update the motor response. Therefore, the "open-loop" motor response cannot be viewed as entirely preplanned. Crossman and Goodeve (1983) and Keele and Posner (1968) proposed a strategy of intermittent correction by a single control mechanism to explain the speed-accuracy relationship described by Fitts (1954). However, no evidence for intermittency was found for any of the observed control mechanisms in the present study. Rather, discontinuities in torque trajectories, when they occurred, demarcated the recruitment of new control mechanisms (Fig. 4).

It is unclear at this point whether the successive approximation strategy can be generalized to single-joint movements, as hypothesized by Greene $(1972,1982)$. The mechanical consequences of single-joint movements are more complex than the production of torque at an isometric joint. Clearly, the control of multijoint limb movement is more complex than the control of joint torque since the movements of the individual joints must be coordinated (Soechting and Lacquaniti, 1981; Lacquaniti and Soechting, 1982). However, it is also possible that complex strategies used to control multijoint movements of the limb are at least partly based on more basic strategies that are used to control single joints.

The results obtained with torque responses to unpredictable targets may be more confidently generalized to everyday motor activities. First, the analysis of responses to completely predictable targets revealed that torque was controlled by the same 3 mechanisms that control responses to unpredictable target. The only apparent difference between responses to predictable and unpredictable targets was the amount of error remaining to be corrected by the first and second adjustments. Second, everyday movements are not always made along entirely predictable trajectories. Situations where the target or the limb is in motion prior to movement onset or where the initial limb or target location is not visible are likely to result in initially inaccurate movements that require subsequent corrective action. These everyday situations are comparable to the experimental paradigm using targets with unpredictable amplitudes.

\section{References}

Beggs, W. D., and C. I. Howarth (1970) Movement in a repetitive motor task. Nature 225: 752-753.

Bizzi, E., and W. Abend (1983) Postural control and trajectory formation in single- and multi-joint arm movements. In Advances in Neurology, Vol. 39, Motor Control Mechanisms in Health and Disease, J. E. Desmedt, ed., pp. 31-45, Raven, New York.

Brown, S. H. C., and J. D. Cooke (1981) Amplitude- and instructiondependent modulation of movement-related electromyogram activity in humans. J. Physiol. (Lond.) 316: 97-107.

Brown, S. H. C., and J. D. Cooke (1984) Initial agonist burst duration depends on movement amplitude. Exp. Brain Res. 55: 523-527.

Burke, R. E., D. N. Levine, P. Tsairis, and F. E. Zajac (1973) Physiological types and histochemical profiles in motor units of the cat gastrocnemius. J. Physiol. (Lond.) 234: 723-748.

Carlton, L. G. (1981) Processing visual feedback information for movement control. J. Exp. Psychol. 7: 1019-1030.

Cooke, J. D., and V. A. Diggles (1984) Rapid error correction during human arm movements: Evidence for central monitoring. J. Motor Behav. 16: 349-363.
Cordo, P. J. (1983) Visually mediated feedback corrections during isometric force tracking in humans. Soc. Neurosci. Abstr. 9: 65.

Cordo, P. J., and L. M. Nashner (1981) Rapid voluntary arm movements in man: Electromyography and performance. Soc. Neurosci. Abstr. 7: 480 .

Crossman, E. R. F. W., and P. J. Goodeve (1963) Feedback control of hand-movement and Fitts Law. Paper presented at the meeting of the Experimental Psychology Society, Oxford, July 1963. Published (1983) in Q. J. Exp. Psychol. 35A: 251-278.

Evarts, E. V., and J. Tanji (1974) Gating of motor cortex reflexes by prior instruction. Brain Res. 71: 479-494.

Favilla, M., W. Hening, and C. Ghez (1985) Human tracking performance: Parallel specification of amplitude and direction. Soc. Neurosci. Abstr. 11(Pt. 1): 72.

Feldman, A. G. (1966a) Functional tuning of the nervous system with control of movement or maintenance of a steady posture. II. Controllable parameters of the muscle. Biophysics 11: 565-578.

Feldman, A. G. (1966b) Functional tuning of the nervous system with control of movement or maintenance of a steady posture. III. Mechanographic analysis of the execution by man of the simplest motor tasks. Biophysics 11: 766-775.

Fitts, P. M. (1954) The information capacity of the human motor system in controlling the amplitude of movement. J. Exp. Psychol. 47: 381-391.

Ghez, C., and J. H. Martin (1982) The control of rapid limb movement in the cat. III. Agonist-antagonist coupling. Exp. Brain Res. 45: 115125.

Ghez, C., and D. Vicario (1978) The control of rapid limb movement in the cat. II. Scaling of isometric force adjustments. Exp. Brain Res. 33: 191-202.

Ghez, C., D. Vicario, J. H. Martin, and H. Yuyima (1983) Sensory motor processing of target movement in motor cortex. In Advances in Neurology, Vol. 39, Motor Control Mechanisms in Health and Disease, J. E. Desmedt, ed., pp. 61-92, Raven, New York.

Greene, P. H. (1972) Problems of organization of motor systems. Prog. Theor. Biol. 2: 303-338.

Greene, P. H. (1982) Why is it casy to control your arms? J. Motor Behav. 14: 260-286.

Hallett, M., B. T. Shahani, and R. R. Young (1975) EMG analysis of stereotyped voluntary movements in man. J. Neurol. Neurosurg. Psychol. 38: 1154-1162.

Hammond, P. H. (1960) An experimental study of servo action in human muscular control. Proc. Conf. Med. Electron., 3rd, London, pp. 190-199.

Hick, W. E. (1952) On the rate of gain of information. Q. J. Exp. Psychol. 4: 11-26.

Keele, S. W. (1968) Movement control in skilled motor performance. Psychol. Bull. 70: 387-403.

Keele, S. W., and M. I. Posner (1968) Processing of visual feedback in rapid movements. J. Exp. Psychol. 77: 155-158.

Kelso, J. A. S., K. G. Holt, and A. E. Flatt (1980) The role of proprioception in the perception and control of human movement: Toward a theoretical reassessment. Perception Psychophys. 28: 45-52.

Kernell, D. (1975) The adaptation and relation between discharge frequency and current strength of cat lumbosacral motoneurons stimulated by long-lasting injected currents. Acta Physiol. Scand. 65: 6773.

Lisberger, S. G., and L. E. Westbrook (1985) Properties of visual inputs that initiate horizontal smooth pursuit eye movements in monkeys. J. Neurosci. 5: 1662-1673.

McCloskey, D. I. (1981) Corollary discharges: Motor commands and perception. In Handbook of Physiology, Vol. 2, The Nervous System, V. B. Brooks, ed., pp. 1415-1447, Williams \& Wilkins, Baltimore, MD.

Megaw, E. D. (1974) Possible modification to a rapid-ongoing programmed manual response. Brain Res. 71: 425-441.

Meinck, H. M., R. Benecke, W. Meyer, J. Hohne, and B. Conrad (1984) Human ballistic finger flexion: Uncoupling of the three-burst pattern. Exp. Brain Res. 55: 127-133.

Milner-Brown, H. S., R. B. Stein, and R. Yemm (1973) Changes in firing rate of human motor units during linearly changing voluntary contractions. J. Physiol. (Lond.) 230: 371-390.

Polit, A., and E. Bizzi (1979) Characteristics of motor programs underlying arm movements in monkeys. J. Neurophysiol. 42: 183-194. 
Poulton, E. C. (1981) Human manual untrol. In Handbook of Physiology, Vol. 2, Motor Control, V. B. Brooks, ed., pp. 1337-1390, Williams \& Wilkins, Baltimore, MD.

Schmidt, R. A., H. Zelaznik, B. Hawkins, J. S. Frank, and J. T. Quinn, Jr. (1979) Motor-output variability: A theory for the accuracy of rapid motor acts. Psychol. Rev. 86: 415-451.

Shae, C. H., J. B. Krampitz, H. Tolson, A. A. Ashby, and R. M. Howard (1981) Stimulus velocity, duration and uncertainty as determiners of response structure and timing accuracy. Res. Q. Exerc. Sport 52 : 86-99.

Shae, C. H., J. B. Krampitz, and C. C. Northam (1982) Information processing in coincident timing tests: A development perspective. J. Hum. Movement Stud. 8: 73-83.
Sittig, A. C., J. J. Denier van der Gon, C. C. A. M. Geilen, and A. J. M. van Wijk (1985) The attainment of target position during steptracking movements despite a shift of initial position. Exp. Brain Res. 60: 407-410.

Smith, W. M., and K. F. Bowen (1980) The effects of delayed and displaced visual feedback on motor control. J. Motor Behav. 12: 91101.

Wachholder, K., and H. Altenburger (1926) Beitrage zur Physiologie der willkurlichen Bewegung. Pfluegers Arch. 9-10:625-661.

Wadman, W. J., J. J. Denier van der Gon, R. H. Geuze, and C. J. Moe (1979) Movements of fast goal-directed arm movements. J. Hum. Movement Stud. 5: 3-17. 